\title{
Perception of Halitosis among General Population
}

Mahnoor Khawaja M. Saleem, Abhishek Lal, Sabeen Masood, Dinaz Ghandhi, Anum Arif Sitai, Naseer Ahmed

\section{ABSTRACT}

Objective: To assess the self-perception of halitosis among general population of Karachi along with the oral hygiene methods used. Secondly, the self-perception of halitosis was compared with predictor variables such as age, gender, level of education, and occupation.

Study design and setting: Cross-sectional survey; a total of 401 participants of Karachi were recruited in September 2020, Pakistan.

Methodology: Using Google Forms, an online questionnaire was developed and consisted of 27 questions regarding demographics and self-perceived halitosis, which was filled by the participants. For the statistical analysis, a multiple linear regression test was used to analyze any significant relation between predictor variables and self-perceived halitosis.

Results: Out of the 401 participants, 168 (41.9\%) were males and 233 (58.1\%) were females. A total of 184 (45.9\%) participants suffered from halitosis, with more prevalence in males. Mostly, $251(62.6 \%)$ participants perceived to have halitosis themselves, with tooth brushing the most common (44.9\%) way to reduce it. About $340(84.8 \%)$ respondents brushed daily, with twice a day selected by $250(62.3 \%)$. The coated tongue was seen by 124 (30.9\%) of participants themselves. Age was found to be significantly associated with self-perceived halitosis.

Conclusion: Halitosis is a frequent problem most prevalent in male gender. It has medical and psychosocial impact. Although in general, people are aware of perceiving bad breath themselves, there is still a need for public awareness campaigns to disseminate more knowledge and management options regarding halitosis. Frequent visits to dental and medical healthcare professionals should be encouraged to mitigate this problem.

Keywords: Awareness, Halitosis, Prevalence, Self-perception.

How to cite this Article:

Saleem MKM, Lal A, Masood S, Ghandhi D, sitai AA, Ahmed N. Perception of Halitosis among General Population. J Bahria Uni Med Dental Coll. 2021; 11(1):35-39 DOI: https://doi.org/10.51985/XMIF8301

This is an Open Access article distributed under the terms of the Creative Commons Attriution Non Commercial Liciense (http:// creativecommons/org/licences/by-nc/4.0) which permits unrestricted non commercial use, distribution and reproduction in any medium, provided the original work is properly cited.

\section{- - - - - - - - - - - - - - - - - - - - - - - -}

\section{INTRODUCTION:}

Halitosis is an unpleasant odor or smell that is emitted from a person's oral cavity. Other terms that are frequently used

- - - - - - - - - - - - -

Mahnoor Khawaja M. Saleem

I Student,

Altamash Institute of Dental Medicine, Karachi,

I Abhishek Lal

Student,

I Altamash Institute of Dental Medicine, Karachi

I Email: abhishekdarshan@yahoo.com

I Sabeen Masood

BDS (Graduated),

I Altamash Institute of Dental Medicine, Karachi

I Dinaz Ghandhi

Associate Professor, Department of Oral and Maxillofacial

Surgery,

I Altamash Institute of Dental Medicine, Karachi

I Anum Arif Sitai

I Demonstrator, Department of Oral Pathology,

I Altamash Institute of Dental Medicine, Karachi

I Naseer Ahmed

Assistant Professor, Department of Community Dentistry,

- Altamash Institute of Dental Medicine, Karach

Received: 06-Nov-2020

Accepted: 24-Dec-2020

I A to denote halitosis is bad breath, fetor oris, or oral malodor. The primary substance that is responsible for causing halitosis is volatile sulfur compounds (VSCs) which are produced by gram-negative anaerobic bacteria (Treponema denticola, Enterobacteriaceae, Prevotella (Bacteroides) melaninogenica, Porphyromonas gingivalis, endodontalis, Prevotella intermedia, Fusobacterium periodonticum) from protein degradation. ${ }^{1}$ Methyl mercaptan and hydrogen sulfide have been the most common substances isolated from malodor of individuals. ${ }^{2}$ Halitosis is divided into three broad categories: factual halitosis, where either physiological or pathological halitosis is present; pseudo-halitosis, where a person thinks he or she might have halitosis although it's not present; and halitophobia, fear of having bad breath. ${ }^{3}$ The factual halitosis is mainly the one that may require attention by the dentist if it is bothersome for the patient. Halitosis is a problem that affects multiple aspects of life such as social, medical, and psychological. About factors that can be associated with the development of halitosis, ${ }^{33}$ categories have been formed which include intra-oral factors, extra-oral factors, and temporary factors, with intra-oral factors being the most common ones. ${ }^{4}$ The main intra-oral factor that leads to the production of malodor is VSCs produced by gram-negative anaerobic bacteria. The yellowish-white coating of the tongue 
has also been well established as an intra-oral factor leading to malodor. ${ }^{5}$ Extra-oral factors include respiratory, gastrointestinal tract problems, sinusitis, diabetes, kidney problems, and sometimes side effects of some drugs. Temporary factors leading to malodor include foods containing onions, garlic, pepper, use of cigarettes, consumption of alcohol, and lastly morning bad breath. Furthermore, common etiologies related to dentistry leading to bad breath include dental plaque, dental caries, reduced salivary flow, and poor periodontal conditions. ${ }^{6}$ Treatment of halitosis primarily depends on the underlying causative factors, which may be provided by the dentist, physician, or psychologist.

Halitosis is a very commonly encountered problem that may not have medical seriousness but has a serious social stigma. ${ }^{7}$ Malodor if a person has, frequently leads to a decrease in self-confidence and avoidance behaviors particularly in social gatherings. Bad breath is commonly associated with a psychiatric disorder such as phobias, depression, worry and this adversely affects one's self-esteem. ${ }^{8}$ Certain agents are commonly used by the general population to reduce or eliminate malodor such as tooth brushing, mouthwash, chewing gums, flossing, etc. Very rarely does the bad breath have any serious medical implications.

Most commonly, a person who does have bad breath may not notice it since they are not aware of the quality of their bad breath. There is a difference between objectively assessing and self-perception of bad breath which can lead to underestimation of the presence of bad breath. A frequently encountered factor regarding bad breath is that people avoid informing a person of having bad breath. Since it is difficult to judge the level of halitosis, some perceive it as nominal whereas some think of it as high enough to avoid social gatherings. Performing better oral hygiene methods have been proven to reduce bad breath and significantly increase one's self-confidence and self-esteem. ${ }^{9}$

Due to the serious social impact of halitosis perceived in generality, knowledge about this entity is important to reduce its impact on one's quality of life. Many times, individuals themselves know about having bad breath with their peers also suggesting it. Most of the time, poor oral hygiene of the population has been the main factor leading to the development of bad breath, with few people with underlying medical disorders. ${ }^{10}$ Lack of knowledge about self-perception of halitosis leads to negligence to correct it. Therefore, from both social, medical, and psychological perspectives, it is necessary to increase the general population's knowledge of self-perception of halitosis which can be a definitive remedy for their concern.

It was aimed to assess the self-perception of halitosis among general population of Karachi along with the oral hygiene methods used. Secondly, the self-perception of halitosis was compared with predictor variables such as age, gender, level of education, and occupation.

\section{METHODOLOGY:}

In this cross-sectional survey, total 401 participants that belonged to Karachi, Pakistan were recruited. The duration of data collection was from 1 Sept 2020 to 30 Sept 2020.

A questionnaire was formulated online using Google Forms, and the generated link was sent to the participants through different social media platforms such as WhatsApp, Emails, Facebook, and Twitter, and their responses were recorded. To calculate sample size, OpenEpi software was used keeping the desired percentile of 50 and a confidence interval of 95 . The calculated sample size for this study was $384(\mathrm{n}=[\mathrm{Z12}-$ á/2.p.q]/d2 $)^{11}$.A written consent was taken from the participants on the questionnaire. Primarily in our study, our focus was on self-perception of halitosis in the general population using validated tools. ${ }^{12}$

Secondarily demographic including age, gender, level of education, and occupation were also assessed. A questionnaire was designed in accordance with another study with some modifications considering the objectives of the current study. ${ }^{12}$

This questionnaire included questions such as whether the person feels he/she has bad breath or not, duration of awareness of bad breath, source of awareness of bad breath, measures to reduce bad breath, dental treatments, underlying systemic diseases which may lead to halitosis, oral hygiene habits, presence of dry mouth, symptoms of current systemic diseases and social habits which includes smoking. In the questionnaire, all these questions were formulated in English language. Adults who were above 18 and residing in Karachi, Pakistan, were part of this study.

Those who were below 18 years old and not residing in Karachi, Pakistan were excluded from the study. The information gathered from the participants through the filled questionnaires were kept anonymous and confidential. Ethics Review Committee of Altamash Institute of Dental Medicine, Karachi has approved this study (AIDM/EC/09/2020/02).

SPSS statistical software version 25 was used. Along with descriptive statistics, a multiple linear regression test was used to analyze any significant relation of the predictor variables, which are age, gender, education, and occupation with Halitosis. A p-value of $<0.05$ was considered to be as statistically significant.

\section{RESULTS:}

In this study, a total of 450 questionnaires were received. On scrutiny, 49 forms were excluded based on being irrelevant and incomplete. A total of 401 participant's data was included in this study. The response rate was calculated to be $89.1 \%$. Descriptive statistics and multiple linear regression tests were used to identify any significant relationship of predictor variables, which were age, gender, level of education, and occupation with Halitosis. Of the 401 participants, the majority of participants, about 222 (55.4\%) belonged to the 
Mahnoor Khawaja M. Saleem, Abhishek Lal, Sabeen Masood, Dinaz Ghandhi, Anum Arif Sitai, Naseer Ahmed

age group of 18-30 years. Among the participants; majority were 233 (58.1\%) female participants. Majority were 43.4\% were graduates. Most of the participants in our study were students $(35.7 \%)$ as shown in table 1 . Regarding questions to assess halitosis, the majority that is $45.9 \%$ (184) of respondents self-perceived bad breath, with $40.9 \%$ (164) participants answering "No" and a few $53(13.2 \%)$ not sure about it. Of the participants suffering from bad breath, most were aware of suffering from bad breath since "Weeks ago" (20.7\%) and "Years ago" (19.7\%). About finding out whether having bad breath or not, mostly 251 (62.6\%) participants figured it out by themselves. Regarding measures opted to reduce bad breath, the majority of participants i.e. $44.9 \%$ chose "Toothbrush" as a measure to reduce bad breath as shown in figure 1 . About $339(84.5 \%)$ participants did not visit the dentist to have an examination performed for bad breath. Furthermore, 359 (89.5\%) respondents did not have an examination performed for conditions that are associated with bad breath. Predominantly, 347 (86.5\%) participants did not have any treatments performed either by a physician or dentist to treat their bad breath. Regarding oral hygiene, most of the $340(84.8 \%)$ participants brushed their teeth regularly with a few $61(15.2 \%)$ did not. About the frequency of brushing per day, 2 times a day was the most commonly select option by $250(62.3 \%)$ number of participants. About flossing, 307 (76.6\%) respondents did not floss their teeth with only a small number of $94(23.4 \%)$ doing it. The majority of 275 (68.6\%) participants did not use mouthwash every day, with those who used mouth wash, 93 (23.2\%) of them using it "Once" a day.

Regarding bleeding gums, 250 (62.3\%) respondents answered "No" when asked about bleeding from gums after brushing teeth. The majority of $313(78.1 \%)$ participants did not have any loose teeth, with a small number of $60(15 \%)$ having loose teeth. Most of the $311(77.6 \%)$ participants did not suffer from dry mouth, few $56(14 \%)$ reported to suffer from it. About suffering from dry eyes, the majority of $338(84.3 \%)$ participants stated not to suffer from dry eyes. Most of the $283(70.6 \%)$ participants did not have a bad taste in their mouths. Regarding the presence of coated tongue, 124 (30.9\%) participants responded "Yes" to have their tongue frequently coated with yellowish or white deposits, with $221(55.1 \%)$ answering "No".

About the medical history of the respondents, 194 (48.4\%) respondents had a positive medical history, with stomach problems $(37.1 \%)$, emotional disturbance $(38.7 \%)$, sinusitis (30.4\%), and diabetes (28.4\%) mainly selected. Regarding problems because of bad breath, the majority of $320(79.8 \%)$ participants did not suffer from any problems due to the presence of bad breath with a small number of 52 (13\%) reported hesitation of other people talking to them. Most of the $309(77.1 \%)$ participants were not smokers. Asking other people other than healthcare professionals to judge bad breath, mostly $331(82.5 \%)$ participants did not ask other people's opinion of their bad breath.

To analyze relation between age, gender, education, and occupation with Halitosis, multiple linear regression test was used with a $p$-value of $=0.05$ considered as significant. A significant relation was found of age $(p$-value $=0.00)$ when compared with halitosis. Although similar was not seen with gender $(p$-value $=0.89)$, education $(p$-value $=0.56)$ and occupation $(\mathrm{p}$-value $=0.12$ ) in relation to halitosis, as shown in table 2.

Table 1: Demographic characteristics of the participants

\begin{tabular}{|c|c|c|}
\hline \multirow{4}{*}{ Age } & Demographic Variables & $\mathbf{n ~ ( \% )}$ \\
\cline { 2 - 3 } & $18-30$ years & $222(55.4 \%)$ \\
\cline { 2 - 3 } & $31-40$ years & $81(20.2 \%)$ \\
\cline { 2 - 3 } Gender & $41-50$ years & $49(12.2 \%)$ \\
\cline { 2 - 3 } & Above 60 years & $32(8.0 \%)$ \\
\cline { 2 - 3 } Education & Male & $17(4.2 \%)$ \\
\hline \multirow{5}{*}{} & Female & $168(41.9 \%)$ \\
\cline { 2 - 3 } & Undergraduate & $233(58.1 \%)$ \\
\cline { 2 - 3 } & Graduate & $150(37.4 \%)$ \\
\hline \multirow{5}{*}{ Occupation } & $174(43.4 \%)$ \\
\cline { 2 - 3 } & Below graduate & $59(14.7 \%)$ \\
\cline { 2 - 3 } & Student & $18(4.5 \%)$ \\
\cline { 2 - 3 } & Healtheare Professional & $82(20.4 \%)$ \\
\cline { 2 - 3 } & Engineer & $24(6.0 \%)$ \\
\cline { 2 - 3 } & Teacher & $27(6.7 \%)$ \\
\cline { 2 - 3 } & Unemployed & $34(8.5 \%)$ \\
\cline { 2 - 3 } & Others & $36(9.0 \%)$ \\
\hline
\end{tabular}

Figure 1: Measures used to reduce bad breath $(n=401)$

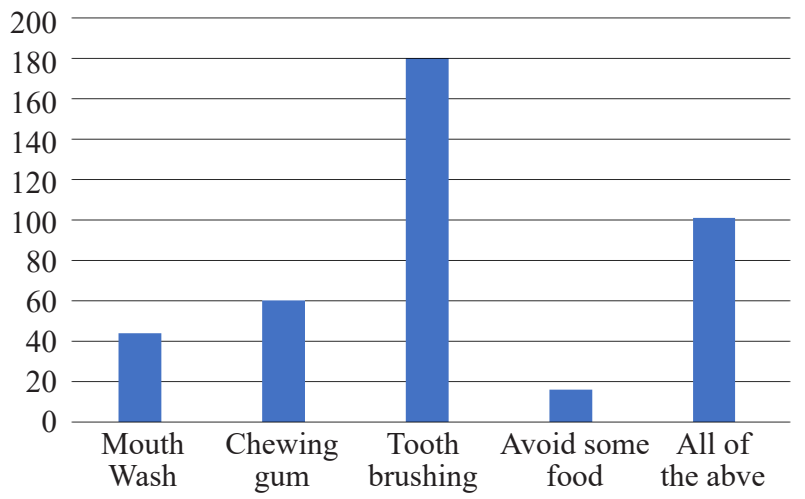

Table 2: Relation of age, gender, education and occupation with Halitosis using multiple linear regression test

\begin{tabular}{|c|c|c|c|c|}
\hline Variables & Coefficient & Standard Error & T-value & p-value \\
\hline Age & -0.254 & 0.233 & -4.290 & 0.001 \\
\hline Gender & 0.007 & 0.481 & 0.137 & 0.891 \\
\hline Education & 0.034 & 0.330 & 0.567 & 0.567 \\
\hline Occupation & 0.089 & 0.130 & 1.555 & 0.121 \\
\hline
\end{tabular}




\section{DISCUSSION:}

Halitosis or Bad breath is a very common problem present worldwide with not only medical consequences but social and psychological as well. The prevalence of halitosis throughout the globe has been strongly established. ${ }^{11}$ In our study, $45.9 \%$ of the participants self-perceived for having bad breath. Previous studies in the literature suggest 8-50\% of the people self-perceived bad breath. ${ }^{13}$

Age has a significant impact on the self-perception of bad breath as reported in our study. The younger adults were more aware of their breath and if it appeared bad, they perceived it. One reason for this could be that younger adults are more vigilant of their breath as they are part of many social gatherings as compared to older adults. ${ }^{14}$ Moreover, this study concluded that males suffered and perceived bad breath more as compared to females. One reason for this could be that females tend to take better care of their oral hygiene as compared to males according to literature. ${ }^{15}$ However, the level of education played no significant role in judging one's bad breath in our study. This contrasts with previous studies that report lower education leads to poor self-perception of halitosis. ${ }^{16}$

Tooth brushing regularly, at least twice a day has been the standard protocol to keep oral hygiene optimum. Majority reported very good oral hygiene with brushing their teeth regularly and twice per day in this study. This, however, may be due to the majority of individuals in the sample belonging to good socioeconomic status, which plays a role in maintaining and performing optimal oral hygiene measures. ${ }^{17}$ If oral hygiene is good and yet halitosis persists in an individual, then the coated tongue is most likely the cause. ${ }^{18}$ This could be the reason individuals in this study reported bad breath despite having good oral hygiene. Although one study did conclude decreased long-term benefit of performing tongue cleaning such as with tongue scrapers. ${ }^{19}$

Most of the respondents in this study did not report to the dentist or physician despite having bad breath. In accordance with Hammad et al., most of the participants tried to solve their bad breath with a measure like chewing gums, mouth wash, and tooth brushing. ${ }^{20}$ This means that most of the participants lack knowledge about halitosis regarding its causes and management plans. A study reports that chewing gums tend to decrease VSCs, thus individuals decrease their self-perception of bad breath. ${ }^{21}$ In a Nigerian study where most of the participants chose a close friend to ask their opinion, these results contrast with our study where most of the individuals themselves evaluated whether they had bad breath or not. ${ }^{22}$ To measure halitosis, the organoleptic score is the gold standard method.

Although rare, some systemic diseases are known to cause bad breath. Conditions like diabetes, tonsillitis, sinusitis, liver problems, and stomach problems are known to cause halitosis. Although this was correlation was not found in our study. Similarly, no correlation was found in our study between the intake of medicines and halitosis, although previous studies do report a positive association. ${ }^{23}$ Furthermore, improper breathing could also result in bad breath. $^{24}$

Smoking was found among 92 participants in this study. It is concluded from previous studies that individuals use smoking as a method to overcome their halitosis problem. ${ }^{25}$ This then leads to a strong smoker's breath, which can be smelled nearby. When patients with halitosis seek treatment for it, they are strongly encouraged to quit it. ${ }^{26}$

Flossing has been an adjunctive method to maintain and improve oral hygiene along with other modalities such as tooth brushing and mouthwashes. In our study, very few individuals used dental floss as part of their routine oral care. Although the importance of dental floss in oral health has been emphasized in literature. ${ }^{27}$

Strengths of this study includes the utility of a validated tool. Whereas, limitations of the study are as bad breath was assessed relying on self-perceived data without clinical examination. Since the questionnaire was in English language so illiterate individuals or people not using online tools got excluded from the study. Lastly, those participants who reported not to have halitosis, it was not clear whether they were free from it or afraid of telling as a result of embarrassment.

\section{CONCLUSION:}

Halitosis is a frequent problem most prevalent in male gender. It has medical and psychosocial impact. Although in general, people are aware of perceiving bad breath themselves, there is still a need for public awareness campaigns to disseminate more knowledge and management options regarding halitosis. Frequent visits to dental and medical healthcare professionals should be encouraged to mitigate this problem.

「 Authors Contribution:

| Mahnoor Khawaja M. Saleem: Literature review and drafted |

I the manuscript

Abhishek Lal: Literature review, drafted the manuscript and I data interpretation

| Sabeen Masood: Drafted the manuscript and performed data analysis

Dinaz Ghandhi: Conducted final and critical review of the manuscript

I Anum Arif Sitai: Drafted the manuscript and collected data

I Naseer Ahmed: Performed final review and approval of the

manuscript

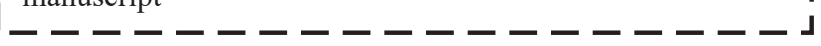

REFERENCES:

1. Rösing CK, Loesche W. Halitosis: an overview of epidemiology, etiology and clinical management. Braz Oral Res [Internet]. 2011;25(5):466-71.

2. Zalewska A, Zatoñski M, Jab ${ }^{3}$ onka-Strom A, Paradowska A, Kawala B, Litwin A. Halitosis--a common medical and social problem. A review on pathology, diagnosis and treatment. Acta Gastroenterol Belg [Internet]. 2012;75(3):300-9. 
3. Özen ME, Aydin M. Subjective Halitosis: Definition and Classification. J N J Dent Assoc [Internet]. 2015;86(4):20-4.

4. Aimetti M, Perotto S, Castiglione A, Ercoli E, Romano F. Prevalence estimation of halitosis and its association with oral health-related parameters in an adult population of a city in North Italy. J Clin Periodontol [Internet]. 2015; 42(12):1105-14.

5. Ashwath B, Vijayalakshmi R, Malini S. Self-perceived halitosis and oral hygiene habits among undergraduate dental students. J Indian Soc Periodontol [Internet]. 2014;18(3):357.

6. Yamunadevi A, Selvamani M, Mohan Kumar K, Basandi P, Madhushankari G. Halitosis - An overview: Part-I Classification, etiology, and pathophysiology of halitosis. J Pharm Bioallied Sci [Internet]. 2015;7(6):339.

7. Cortelli JR, Barbosa MDS, Westphal MA. Halitosis: a review of associated factors and therapeutic approach. Braz Oral Res [Internet]. 2008;22(suppl 1):44-54.

8. Sedky NA. Perceived Impact of Halitosis on Individual's Social life and Marital Relationship in Qassim Province, KSA. J Am Sci [Internet]. 2015;11(3).

9. Setia S, Pannu P, Gambhir R, Galhotra V, Ahluwalia P, Sofat A. Correlation of oral hygiene practices, smoking and oral health conditions with self perceived halitosis amongst undergraduate dental students. J Nat Sci Biol Med [Internet]. 2014;5(1):67.

10. Ziaei N, Hosseinpour S, Nazari H, Rezaei M, Rezaei K. Halitosis And Its Associated Factors Among Kermanshah High School Students (2015). Clin Cosmet Investig Dent [Internet]. 2019;11:327-38.

11. Bakhshi M, Tofangchiha M, Bakhtiari S. Prevalence of selfperceived halitosis, demographic factors and oral health care among defined groups of dental students in Iran. J Int Oral Heal [Internet]. 2018;10(4):187.

12. University of British Columbia Breath Testing Clinic. J Can Dent Assoc [Internet]. 66(5). Available from: https://www.cdaadc.ca/jcda/vol-66/issue-5/259.pdf

13. Porter SR, Scully C. Oral malodour (halitosis). BMJ [Internet]. 2006;333(7569):632-5.

14. Alzoubi FQ, Karasneh JA, Daamseh NM. Relationship of psychological and oral health statuses with self-perceived halitosis in a Jordanian population: a cross-sectional study. BMC Oral Health [Internet]. 2015;15(1):89.
15. Mamai-Homata E, Koletsi-Kounari H, Margaritis V. Gender differences in oral health status and behavior of Greek dental students: A meta-analysis of 1981, 2000, and 2010 data. J Int Soc Prev Community Dent [Internet]. 2016;6(1):60.

16. Al-Ansari JM, Boodai H, Al-Sumait N, Al-Khabbaz AK, AlShammari KF, Salako N. Factors associated with self-reported halitosis in Kuwaiti patients. J Dent [Internet]. 2006;34(7): 444-9.

17. Alsadhan S DA. Non-oral factors associated with self-reported halitosis among adults living in Riyadh, Saudi Arabia. OHDM [Internet]. 2015;14(1):58-61.

18. Gonçalves AC de S, Martins MCN, Paula BL de, Weckwerth $\mathrm{PH}$, Franzolin S de OB, Silveira EMV. A new technique for tongue brushing and halitosis reduction: the $\mathrm{X}$ technique. $\mathrm{J}$ Appl Oral Sci [Internet]. 2019;27. Available from: http://www.scielo.br/scielo.php? script=sci_arttex t\&pid= S1678-7 7572019000100437\&tlng=en

19. Outhouse TL, Al-Alawi R, Fedorowicz Z, Keenan J V. Tongue scraping for treating halitosis. In: Outhouse TL, editor. Cochrane Database of Systematic Reviews [Internet]. Chichester, UK: John Wiley \& Sons, Ltd; 2006. Available from: http://doi.wiley.com/10.1002/14651858.CD005519.pub2

20. Tarakji B, Al-Waeli H, Alhadithy T, Darwazeh A, Hammad M. Prevalence and awareness of halitosis in a sample of Jordanian population. J Int Soc Prev Community Dent [Internet]. 2014;4(6):178.

21. De Luca-Monasterios F, Chimenos-Küstner E, López-López J. Efecto de masticar chicle sobre la halitosis. Med Clin (Barc) [Internet]. 2014;143(2):64-7.

22. Afolabi AO, Ogundipe OK EG. Halitosis (bad breath): an assessment of the awareness and knowledge of health providers in a Nigerian community. Sudan Med J [Internet]. 2009; 45:43-8.

23. Torsten M, Gómez-Moreno G, Aguilar-Salvatierra A. Drugrelated oral malodour (halitosis): a literature review. Eur Rev Med Pharmacol Sci [Internet]. 2017;21(21):4930-4.

24. Bollen CM, Beikler T. Halitosis: the multidisciplinary approach. Int J Oral Sci [Internet]. 2012;4(2):55-63.

25. Tangerman A. Halitosis in medicine: A review. Int Dent $\mathrm{J}$ [Internet]. 2002;52(S5P1):201-6.

26. Rosenberg M. Clinical Assessment Of Bad Breath: Current Concepts. J Am Dent Assoc [Internet]. 1996;127(4):475-82.

27. Aylikci B, Çolak H. Halitosis: From diagnosis to management. J Nat Sci Biol Med [Internet]. 2013;4(1):14. 